\title{
Improving Slip Prediction on Mars Using Thermal Inertia Measurements
}

\author{
Christopher Cunningham and William L. Whittaker \\ The Robotics Institute \\ Carnegie Mellon University \\ Email: $\{$ ccunningham, red $\} @$ cmu.edu
}

\author{
Issa A. Nesnas \\ Jet Propulsion Laboratory \\ California Institute of Technology \\ Email: issa.a.nesnas@jpl.nasa.gov
}

\begin{abstract}
Rovers operating on Mars have been delayed, diverted, and trapped by loose granular materials. Vision-based mobility prediction approaches often fail because hazardous sand is difficult to distinguish from safe sand based on surface appearance alone. Unlike surface appearance, the thermal inertia of terrain is directly correlated to the same geophysical properties that control slip. This paper presents a quantitative analysis showing that considering thermal inertia improves rover slip prediction on Mars using in-situ data from the Curiosity rover. Thermal inertia is estimated for each slip measurement in sand using both on-board and orbital instruments. Slip models are learned using a mixture of experts approach where the experts are identified using thermal inertia. Two-expert models are compared to a single-expert, vision-only model to show that slip predictions are improved by separating high-slip, low thermal inertia sand from low-slip, high thermal inertia sand. These results support the hypothesis that the consideration of thermal inertia improves mobility estimates for rovers on Mars.
\end{abstract}

\section{INTRODUCTION}

The most significant mobility challenges that rovers have encountered on Mars have been caused by loose, granular materials. Sandy regions cause wheels to slip and sink, which slows progress and risks entrapping a vehicle. However, terrain on Mars is very diverse. Both the macroscopic and microscopic characteristics of sand varies greatly over the surface. The inherent difficulty of predicting slip is exacerbated by the risk-averse nature of planetary rover missions that avoid sand regions whenever possible. This results in few training examples from which to learn a competent prediction model. Consequently feature-intensive, visual approaches that only consider surface appearance risk significant over-fitting. Instead, this paper uses thermal inertia as a single measurement that is highly correlated to the underlying granular properties that govern wheel-terrain interaction.

Loose, granular terrain has caused mobility problems for Curiosity and the Mars Exploration Rovers, Spirit and Opportunity. All three rovers have suffered significant delays caused both by excessive slip in hazardous sand and time wasted analyzing safe sand before successful traversal [4, 50]. The most serious mobility problem was encountered by Spirit when its wheels fell through a thin cemented surface layer (duricrust) into weak, undetected sand. The sand entrapped the rover until it eventually stopped responding to commands [3]. Means to quickly and accurately evaluate traversability of terrain before contact would significantly increase the safety and operational cadence of rovers on Mars.

Thermal inertia is a physical property of a material that represents its resistance to changes in temperature and can be indirectly estimated by fitting an analytical model to observations of surface temperature. In a granular material, thermal inertia is strongly influenced by many of the same physical characteristics that influence wheel terrain interaction, which suggests that thermal inertia measurements could improve predictions of traversability [9]. Recent work has used this relationship to distinguish loose and compact granular materials both experimentally on Earth [12, 13] and in simulation on the Moon [14]. Unfortunately, due to differences in geology, gravity, and atmospheric pressure, showing a correlation between thermal inertia and mobility on Earth and the Moon is not a guarantee that the same correlation exists on Mars. There is some evidence that thermal inertia is correlated to traversability on Mars as well [38]; however, to the best of the authors' knowledge, no study has investigated its ability to improve mobility predictions using in-situ rover data.

This paper analyzes the potential of thermal inertia measurements to improve slip prediction in Martian sand using in-situ data from the Curiosity rover. For each wheel slip measurement in sand, thermal inertia is estimated using both Curiosity's onboard Ground Temperature Sensor (GTS) and the orbital Thermal Emission Imaging System (THEMIS). A mixture of experts (MoE) approach is used to model slip behavior. This model assumes that slip cannot be explained by a single type of sand but is instead a combination of multiple independent models. A soft-max function assigns data to experts using thermal inertia as its input. Results from twoexpert models are compared against a single-expert, visiononly model to show the ability of thermal inertia to identify high-slip sand regions using both ground-based and orbital observations.

This paper is organized as follows. Section III discusses related work in slip prediction. Section III describes the connection between thermal inertia and mobility. Section IV outlines how thermal inertia is estimated and slip models are learned. Section $\mathrm{V}$ describes the dataset from Curiosity used in this analysis. Analysis of slip models and the efficacy of thermal inertia is discussed in Section VI Section VII discusses conclusions and directions for future work. 


\section{RELATED WORK}

The vast majority of traversability prediction approaches use either vision, geometric sensing, or a combination of both. For the Mars rovers, operators predict slip by first identifying terrain class visually from Navcam imagery then estimating slip from average terrain slope using Earth-calibrated slip versus slope curves [24]. Recent work on Curiosity has improved this process for Curiosity using a CNN-based classifier to visually classify terrain and estimating slip using models learned from in-situ experiences on Mars [48]. However, this approach still fails to accurately predict slip in sand. Slip behavior instead appears to be a function of multiple types of sandy terrain. Cunningham et al. [15] use spatial correlations to improve slip prediction in sand. However, this approach still requires proprioceptive measurements of slip and thus cannot detect differences in sand traversability before contact.

The rovers also have on-board autonomy and safeguarding for mobility. When operating autonomously, they use the Grid-based Estimation of Surface Traversability Applied to Local Terrain (GESTALT) algorithm to avoid rough terrain and geometric hazards; however, this does not address slip prediction [19]. In addition, due to computational cost, the rover is rarely commanded to navigate autonomously. Instead, most rover traverses are designed by rover operators and drive blindly with the exception of safeguarding by VO-based slip detection [32]. When the VO system predicts that slip has exceeded an operator-defined threshold the drive is stopped and the rover waits for direction from Earth [33].

Terrestrial long-range slip prediction research has also primarily used visual and geometric sensing techniques. Halatci et al. [22] developed a terrain classifier with color, texture, and geometric features to identify rocky, sand, and mixed terrain in Mars rover imagery. Brooks and Iagnemma [7] expanded upon this classifier to instead predict coefficient of traction with a multi-class SVM. Angelova et al. used a mixture of experts approach to first identify terrain classes from imagery then predict slip given the terrain class. Terrain classes were learned from manually labeled imagery and slip was learned from experience using locally weighted projection regression [2, 1]. This paper follows a mixture of experts approach as well, but the classes are instead determined by thermal inertia and learned without supervision. Otsu et al. [37] demonstrated improved visual terrain classification with sparse labeling by co-training with a vibration-based classifier but did not address slip prediction. Ho et al. [25] recently used Gaussian Processes to estimate traversability in deformable terrain based on geometry alone but did not test in sandy regions. All of these approaches suffer from the same shortcoming; they only see macroscopic surface appearance.

In contrast to visual imagery, thermal measurements provide information about the bulk mechanical properties both at and below the surface [45]. This correlation has been used to infer global geologic composition from orbit on Earth [43], the Moon [51, 5], and Mars [18, 35]. Orbital thermal inertia analysis has been used in landing site selection for rovers and is used in long-range strategic traverse planning [20, 16]. Unfortunately, the resolution of orbital thermal imaging sensors is too low to detect many rover-scale hazards. The Miniature Thermal Emission Spectrometer (Mini-TES) instruments aboard the Mars Exploration Rovers and the GTS on Curiosity have been used to analyze surface physical properties but were not designed for traversability prediction and are not used in daily path planning [23, 17, 34]. This paper presents a quantitative analysis of whether considering thermal inertia improves slip prediction on Mars using data from Curiosity's GTS [49] and the Mars Reconnaissance Orbiter's THEMIS [11].

\section{Relating Thermal Inertia AND Mobility}

Thermal inertia is a characteristic of a material that governs its resistance to changes in temperature. Thermal inertia is of interest in this work because of its correlation to mobility. This section presents background on thermal inertia, the geophysical properties that govern it, and its importance to mobility.

\section{A. Analytical Surface Temperature Model}

Here a thermal model is presented in the context of Mars at equatorial to mid-latitudes without frost following the derivation in Putzig [44]. Both the surface and subsurface temperature of homogeneous terrain is governed by the wellknown, semi-infinite one-dimensional heat diffusion equation.

$$
\frac{\partial T}{\partial t}=\frac{k}{\rho c} \frac{\partial^{2} T}{\partial Z^{2}}
$$

where $k$ is thermal conductivity, $\rho$ is bulk density, $c$ is specific heat capacity, $Z$ is the distance into the terrain, and $t$ is time.

The lower boundary condition is usually assumed to be either insulating or a constant geothermal heat flux. The surface boundary condition at $Z=0$ is balance of heat fluxes from the Sun, the atmosphere, and the ground.

$$
(1-A) R_{s w}+\varepsilon R_{l w}-\varepsilon \sigma T_{s}^{4}=-\left.I \sqrt{\frac{\pi}{P}} \frac{\partial T}{\partial Z^{\prime}}\right|_{Z^{\prime}=0}
$$

where $A$ is albedo, $\varepsilon$ is emissivity, $\sigma$ is the Stefan-Boltzmann constant, $P$ is the period of a diurnal cycle, $R_{s w}$ is shortwave radiation from the Sun, $R_{l w}$ is longwave radiation emitted by the atmosphere, $T_{s}$ is the surface temperature, and $\left.\frac{\partial T}{\partial Z^{\prime}}\right|_{Z^{\prime}=0}$ is the temperature gradient at the surface. Let $Z^{\prime}=Z / \delta$ where $\delta$ is the thermal skin depth defined by $\delta^{2}=\frac{k}{\rho c} \frac{P}{\pi}$. At $Z=\delta$ the temperature wave is attenuated by a factor of $e^{-1}$ [44].

The most important term in Equation 2 is $I$, which represents the thermal inertia. Thermal inertia is defined as:

$$
I=\sqrt{k \rho c}
$$

with units of $J m^{-2} K^{-1} s^{-\frac{1}{2}}$ [44]. A change of variables in Equation 1 from $Z$ to $Z^{\prime}$ results in:

$$
\frac{\partial T}{\partial t}=\frac{\pi}{P} \frac{\partial^{2} T}{\partial Z^{\prime 2}}
$$

This isolates all of the terrain properties in $I$ in Equation 2 Hence under periodic illumination, with known surface heat 
fluxes and albedo, thermal inertia controls the surface temperature. Thermal inertia can be estimated by fitting observed surface temperatures to an analytical model [35]. For more detailed derivations see [44] and [28].

\section{B. Thermal Inertia}

Thermal inertia is a function of the thermal conductivity, heat capacity, and density of a material. In a granular material, heat capacity is not strongly affected by density or particle size, varying by factors of no greater than 2 or 3 on Mars. Variations in density directly affect thermal inertia as defined in Equation 3 . However, density varies by less than a factor of 2 in sandy materials. The most significant factor affecting thermal inertia is thermal conductivity [44].

In granular materials, bulk thermal conductivity is determined by three different heat transfer mechanisms: (1) heat conducted through gas in the void space, (2) radiative heat transfer, and (3) solid path heat conduction [39]. The most significant heat transfer path is by gas conduction. However, this is strongly affected by atmospheric pressure [42]. In part because gas conduction dominates in both cases, differences in pressure make it difficult to ensure that terrestrial results will translate to Mars. Radiative heat has only a minor affect. Solid path heat conduction between grains is more significant but is limited by the small grain contacts. The effect is that thermal inertia is not a strong function of the mineralogical composition of a granular material but rather the physical properties of its grains [27]. This is critical for this work since traversability is dependent on physical properties as well.

At Martian pressure, thermal inertia in a granular material is a function of its particle size, density, and cementation. An increase in particle size greatly increases conductivity, which in turn greatly increases thermal inertia [42]. An increase in density also causes a slight increase in thermal conductivity [41]. However, since thermal inertia is a direct function of density as well, it is still significant. Lastly, an increase in cementation causes corresponding increases in thermal conductivity and thermal inertia [40]. Apparent thermal inertia is also strongly affected by layering of materials. A thin layer of sand on top of bedrock will have much higher thermal inertia than very deep sand [45]. See Piqueux and Christensen [39] for an in depth discussion of thermal inertia.

\section{Relation to Mobility}

For a given rover, wheel-terrain interaction in a loose granular material is strongly influenced by particle size and density [8]. Having a larger distribution of particle sizes with larger particles increases soil strength [26, 36]. Similarly, denser, more compact sands are more traversable than fine, loose sands [54, 52]. In addition, as particles become cemented together the terrain becomes more cohesive and, thus, easier to traverse [29, 53]. Therefore, because particle size, density, and cementation strongly influence both traversability and thermal inertia, there is a strong correlation between traversability and thermal inertia. Similarly, as depth of surface sand increases thermal inertia [44] and traversability [4] both decrease.
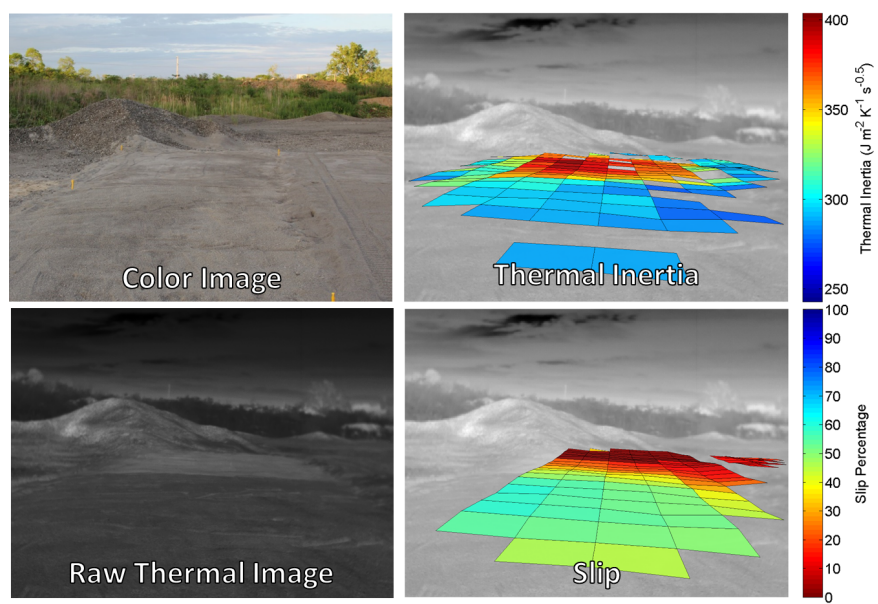

Fig. 1: Transition from loose to compact sand regions shown in color and thermal data. Loose regions were manually loosened with a shovel, and compact regions were compacted with a vibratory compactor. Overlaid plots of estimated nighttime thermal inertia and measured slip are shown on the right. Missing grid cells in the thermal inertia image corresponds to regions with significant height variance.

A correlation between the thermal properties of granular terrain and traversability has been demonstrated experimentally on Earth [13, 12]. A qualitative example is shown in Figure 1. In this example, loose and compact granular materials are difficult to distinguish in visual imagery but are evident in the nighttime thermal image. Thermal inertia was estimated using the model described in Cunningham et al. [12] with ground truth measurements of solar and atmospheric heat flux. A four wheeled rover was driven over the surface while dragging a weighted sled to induce slip. Slip was estimated by comparing ground truth position from a total station to wheel odometry. Slip at a given location was averaged over several drives while avoiding previous wheel tracks. Figure 1 provides a visual of the strong connection between thermal inertia and mobility on Earth. There is also a clear correlation in the nighttime thermal image, which can illuminated relative difference in thermal inertia within an image. However, using thermal inertia models rather than raw imagery enables comparisons across times of day, seasons, and atmospheric conditions. Unfortunately, because atmospheric pressures are very different, a correlation between thermal inertia and traversability on Earth does not guarantee a corresponding correlation on Mars.

In this work, wheel slip will be used as a measure of traversability. Percent slip is defined as:

$$
y= \begin{cases}\left(1-\frac{v}{v_{\text {ref }}}\right) \times 100, & v<v_{\text {ref }} \text { (driving) } \\ \left(\frac{v_{\text {ref }}}{v}-1\right) \times 100, & v>v_{\text {ref }} \text { (braking) }\end{cases}
$$

where $v$ is the rover's velocity and $v_{\text {ref }}$ is the commanded velocity. Slip is defined for $-100 \% \leq y \leq 100 \%, y>0$ indicates that the rover's speed is slower than commanded, and $y<0$ indicates that is is faster than commanded. 


\section{Slip Prediction with Thermal Inertia Classes}

This section describes an approach for that uses thermal inertia to improve slip prediction. Thermal inertia is estimated for each slip measurement with data from Curiosity's GTS. Slip models are then learned using a mixture of experts (MoE) model that automatically separates terrain classes by thermal inertia.

\section{A. Thermal Inertia Estimation}

Thermal inertia is estimated for each slip measurement in sand. Curiosity is equipped with the Ground Temperature Sensor (GTS) that measures ground temperatures for scientific investigation of the geologic composition and atmospheric processes on Mars. The GTS is a set of thermopiles that are located on Curiosity's mast with a fixed pointing at $120^{\circ}$ clockwise off azimuth and $22^{\circ}$ down. It has a horizontal field of view of $60^{\circ}$ and a vertical field of view of $40^{\circ}$. It measures one temperature value from an ellipse of nominally 100 $\mathrm{m}^{2}$ at 4 o'clock relative to the rover's forward direction. This region is adjacent to and not in the path of the rover during straight drives. The baseline mode of operation is measuring temperature at $1 \mathrm{~Hz}$ for about 6 minutes at the beginning of every hour [49]. This is not an ideal sensor for mobility prediction because: (1) it only records one measurement from the entire ellipse and (2) it does not measure the terrain ahead of the rover. However, it still provides an unprecedented amount of data, which enables this investigation.

The KRC model was used to simulate surface temperatures [28]. Given a set of input parameters, it estimates the surface heat fluxes in Equation 2 and forward simulates the heat diffusion equation (Equation (4) to find the surface temperature at a desired time of day. Forward simulation of both surface and subsurface temperatures is accomplished using an iterative finite-differences approach. This model has been used extensively for orbital thermal analysis [18] and recently for GTS data [23]. For more detail see Kieffer [28].

The KRC model requires a number of input parameters to generate predicted surface temperatures. Thermal inertia and albedo are treated as free parameters. Slope, slope azimuth, dust opacity, solar longitude (season), latitude, and elevation were fixed inputs to the model. Slope and slope azimuth were estimated from Navcam imagery. Dust opacity was estimated by scaling Opportunity dust opacity measurements to match the elevation for Curiosity [31]. Opportunity data was used because it is a good approximation and dust opacity data for Curiosity is not yet published [30].

First the GTS data is filtered to remove measurements during which the rover is shadowing the measurement, the measurement falls out of calibration range, or the power supply is out of nominal operating range. Remaining GTS data points are low-pass filtered to reduce noise and then binned into 5 minute intervals throughout the day.

Both thermal inertia and albedo are unknown a priori and estimated following the example of Hamilton et al. [23] by minimizing the squared error between GTS-measured temperatures and the KRC analytical model. Albedo can be roughly

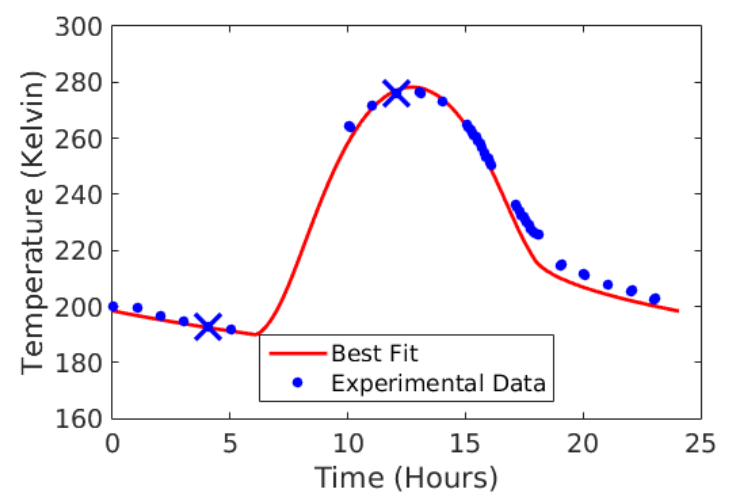

Fig. 2: Example GTS data and best-fit model. The Xs indicate data points used to find the thermal inertia and albedo.

estimated from camera imagery but does not always match thermally-derived albedo [17]. Thus to avoid errors in thermal inertia estimates, albedo is also considered a free parameter. The optimization uses only GTS temperatures from pre-dawn and mid-day because the diurnal amplitude is strongly affected by thermal inertia and using only these measurements reduces error caused by slope and albedo. An example fit is shown in Figure 2 When fitting to only these points, the analytical model underestimates morning and afternoon temperatures, which is likely due to terrain heterogeneity [23]

\section{B. Learning Slip Models}

A two-expert MoE model is used to predict slip and separate low-slip sand from high-slip sand. A fairly simple model with few hyperparameters is used due to the low availability of data. Here it is assumed that slip, $y$, in a given type of sandy terrain, $T_{i}$, is a function of the surface geometry, $x$. Let $\mathbf{x}, \mathbf{y}$, and $\mathbf{I}$ be $K \times 1$ vectors, where $K$ is the number of data points. Additionally, it is assumed that each of $N=2$ terrain classes can be separated using thermal inertia, $I$. This results in the following model for slip in sand:

$$
p(\mathbf{y} \mid \mathbf{I}, \mathbf{x}, \Theta)=\sum_{i=1}^{N} \pi_{i}\left(\mathbf{I}, v_{i}\right) p\left(\mathbf{y} \mid \mathbf{x}, \theta_{i}\right)
$$

where $\pi_{i}\left(\mathbf{I}, v_{i}\right)$ is the gating function for sand class $T_{i}$ with parameters $v_{i}, p\left(\mathbf{y} \mid \mathbf{x}, \theta_{i}\right)$ is the distribution of slip in a given class with parameters $\theta_{i}$, and $\Theta=\left\{\left\{v_{i}\right\}_{1}^{N},\left\{\theta_{i}\right\}_{1}^{N}\right\}$.

The gates are linear softmax functions:

$$
\pi_{i}\left(\mathbf{I}, v_{i}\right)=\frac{\exp \left(v_{i}^{T}[\mathbf{I}, \mathbf{1}]\right)}{\sum_{j=1}^{N} \exp \left(v_{j}^{T}[\mathbf{I}, \mathbf{1}]\right)}
$$

where each $v_{i}$ is a $2 \times 1$ weight vector. This simple softmax function was chosen to essentially provide a threshold on thermal inertia that separates low-slip and high-slip sand. Slip is modeled as an $M^{\text {th }}$ degree polynomial of slope, $\mathbf{y}=\phi(\mathbf{x}) \beta+\varepsilon$. $\beta$ is an $(M+1) \times 1$ weight vector, $\phi(\mathbf{x})=\left[\mathbf{x}^{n}, \mathbf{x}^{n-1}, \ldots, \mathbf{x}^{1}, \mathbf{1}\right]$, and $\varepsilon \sim \mathscr{N}(0, \sigma)$. If $\theta_{i}=\left\{\beta_{i}, \sigma_{i}\right\}$, it follows that

$$
p\left(\mathbf{y} \mid \mathbf{x}, \theta_{i}\right)=p\left(\mathbf{y} \mid \mathbf{x}, \beta_{i}, \sigma_{i}\right)=\mathscr{N}\left(\phi(\mathbf{x}) \beta_{i}, \sigma_{i}\right)
$$


To reduce overfitting, slip is constrained to never decrease with increasing slope [15]. A linear model was chosen because conditioned on the model parameters, the data points are independent and identically distributed (i.i.d.), which makes fitting a MoE model easier than with a nonparametric function [47]. Expectation Maximization is used to find the parameters $\Theta$ for the model following the approach in Yuksel et al. [55]:

(1) E-step. At iteration $m$, compute expected class assignments for each sample $t$ given by $\left\{y_{t}, x_{t}, I_{t}\right\}$ [6]:

$$
h_{t}^{(m)}\left(y_{t} \mid x_{t}, I_{t}\right)=\frac{p\left(y_{t} \mid x_{t}, I_{t}, \Theta_{i}^{(m)}\right)}{\sum_{j=1}^{N} p\left(y_{t} \mid x_{t}, I_{t}, \Theta_{j}^{(m)}\right)}
$$

(2) M-step. Given the expected values for the latent class assignments, compute model parameters $\Theta^{(m+1)}$ :

$$
\begin{gathered}
v^{(m+1)}=\underset{v^{\prime}}{\operatorname{argmax}} \sum_{t=1}^{K} h_{t}^{(m)} \log \pi\left(I_{t}, v^{\prime}\right) \\
\theta^{(m+1)}=\underset{\sigma^{\prime}, \beta^{\prime}}{\operatorname{argmax}} \sum_{t=1}^{K} h_{t}^{(m)} \log p\left(y_{t} \mid x_{t}, \beta^{\prime}, \sigma^{\prime}\right)+\lambda \beta^{\prime T} \beta^{\prime}
\end{gathered}
$$

Where $\lambda$ is a regularization parameter to prevent overfitting. Note that unlike in standard regression models, Equation 11 must be solved using constrained optimization due to the nondecreasing constraint. The $\mathrm{E}$ and $\mathrm{M}$ steps are alternated and repeated until convergence. The only design parameters are $\lambda$ and $M$. $\lambda$ was set to 1 . $M$ was set to 5 because a fifth degree polynomial was the simplest polynomial able to fit slip curves for the non-sand terrain classes shown in Figure 3

\section{TeSting Dataset From CURIOSITY}

This section describes the data from Curiosity. Curiosity is a six wheeled rover with a rocker-bogie suspension system. It is $3 \mathrm{~m}$ long, $2.8 \mathrm{~m}$ wide, and has wheels $0.5 \mathrm{~m}$ in diameter [21]. It has been operating on Mars since August, 2012 and has driven over $15 \mathrm{~km}$. Samples from sols 1-986 are considered here, where a sol refers to a day on Mars. Sand slip measurements are identified and matched to thermal inertia measurements. Resampling occurs to reduce data imbalance.

\section{A. Finding Slip Measurements in Sand}

To identify slip measurements in sand, a list of all VO slip measurements was collected. Most drives with VO enabled are between 0.5 and $1 \mathrm{~m}$ in length. Only drives greater than or equal to $0.5 \mathrm{~m}$ in length are considered to reduce measurement noise. The slip for a given drive is calculated as the average of all individual wheel slips. This slip magnitude includes both longitudinal and lateral slip. Slope is the magnitude of rover tilt averaged over the drive as calculated by the IMU. Positive slope corresponds to driving up the slope and negative slope corresponds to driving down the slope regardless of driving direction (i.e. forward versus reverse). Slip and slope magnitude were used instead of the longitudinal and lateral

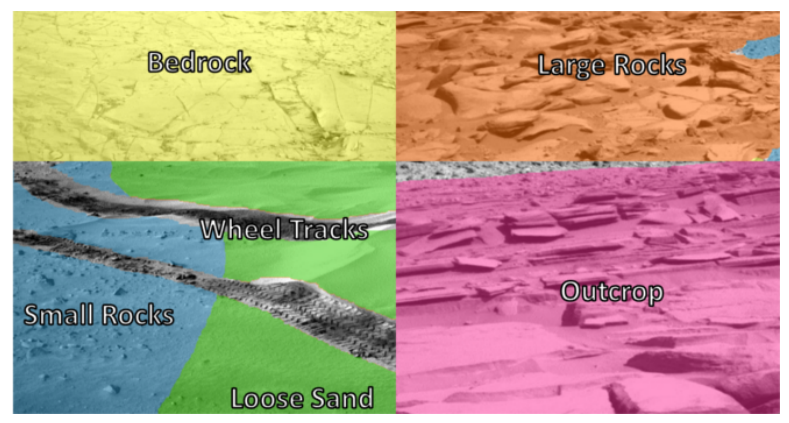

Fig. 3: Six terrain classes identified by SPOC-G classifier [48]

components to reduce the risk of overfitting. Both slip and slope were predominantly in the longitudinal direction.

Manually identifying which drives through sand would likely lead to bias and inconsistencies in the data. Instead, drives through sand were automatically identified using a terrain classifier and false positives are manually removed. Terrain classes are identified by applying the SPOC-G [48] terrain classifier to Navcam imagery. Figure 3 shows the six terrain classes SPOC-G identifies.

$\sim 2500$ Navcam images were classified. The terrain class for each slip measurement was estimated as the mode class of all predictions from all images viewing that location. Using the combination of multiple labeled images for a given location tends to correctly identify more of the drives in sand; however, it also leads to more false positives. This is in contrast to identifying class from only one "best" image as in Rothrock et al. [48], which has fewer false positives but identifies fewer of the actual drives in sand. For example, in Figure 4a, wheel tracks in deep sand were predicted to be outcrops in the closest images but were correctly identified using multiple viewpoints.

Once all sandy regions were automatically identified, false positives were manually filtered out. Measurements where fewer than four of the wheels were on sandy terrain were removed. Examples of correctly identified drives in sand are shown in Figures 4a 4d Examples of false positives that were manually removed are shown in Figures $4 \mathrm{e}$ and $4 \mathrm{f}$. At times it can be difficult to determine exactly what "sand" is. For example, in Figure 4f all of the wheels were on sand. However, under four of the wheels were also rocks on top of the sand, so these samples were removed.

\section{B. Matching Thermal Inertia}

To reduce bias for geologic investigation, Curiosity's GTS points off to the side of the rover towards a region that the rover will never drive over without turning. Thus, slip measurements rarely overlap with GTS thermal inertia measurements. Instead, thermal inertia for a given slip sample was estimated by taking the closest sample that shares the same terrain class as the slip sample (i.e. the closest thermal inertia measurement classified as sand).

The terrain class of each thermal inertia measurement was estimated by first finding the region that the sensor was viewing. Then the terrain class was found using the same approach 


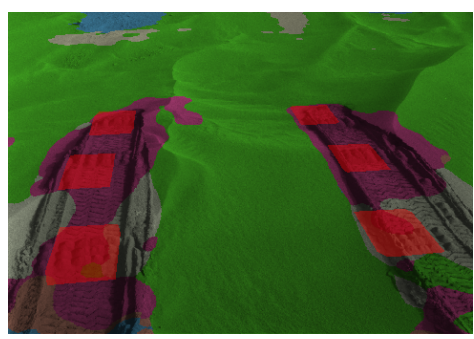

(a) Moosiluake Valley.

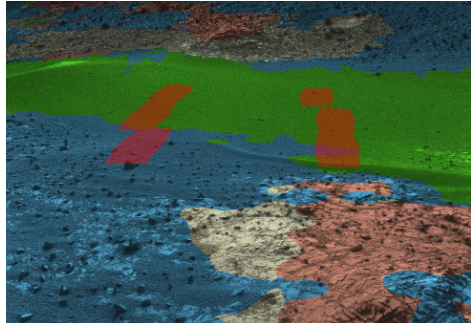

(d) Artists Drive.

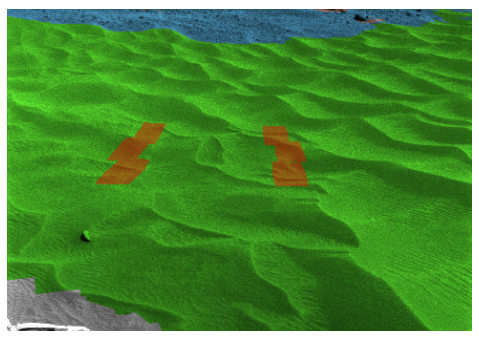

(b) Hidden Valley.

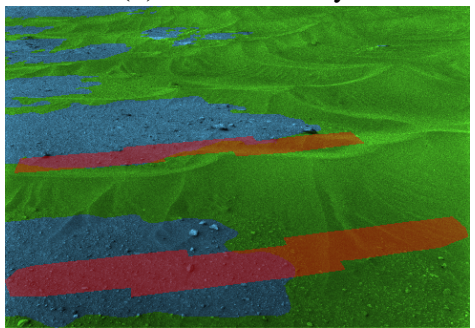

(e) Sol 799 sand.

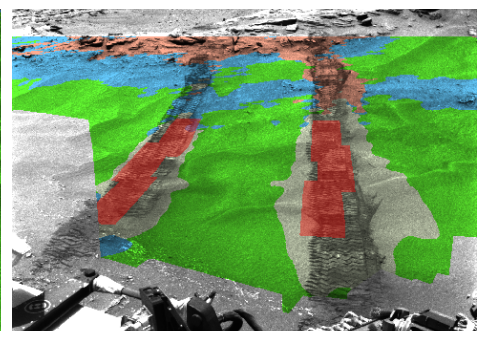

(c) Jubilee Pass.

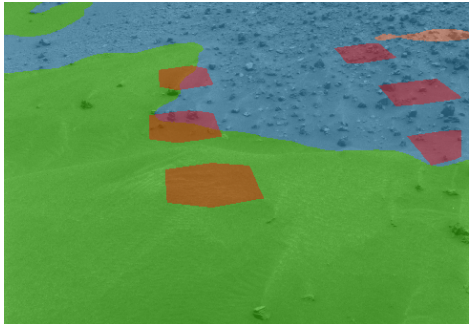

(f) Logan's Pass.

Fig. 4: Example images of slip measurements in sand colorized by predicted terrain class. (a)-(d) were used in the data set, (e) and (f) are examples of measurements that were filtered out due to heterogeneous terrain. The red regions indicate the location of the rover's wheels during its drive.

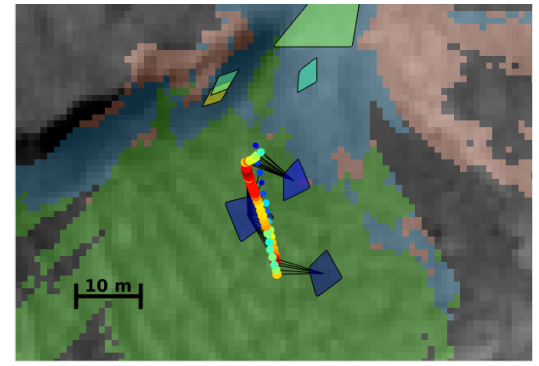

(a) Hidden Valley.

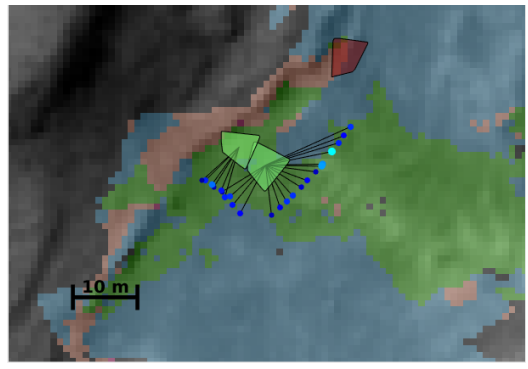

(b) Sol 606 Ripple Crossing.

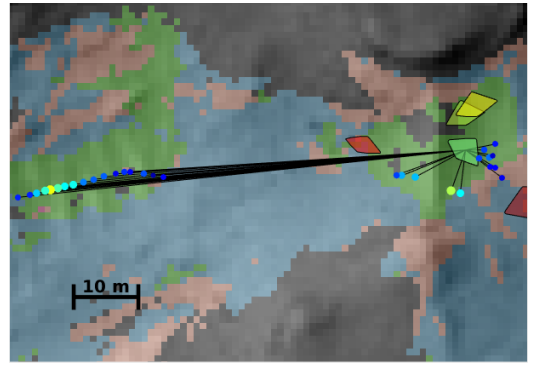

(c) Moonlight Valley (left) \& Dingo Gap.

Fig. 5: Example matches between GTS data and slip data. Slip data points are shown as circular data points at the location of the center of the rover. Data points are sized and colored by slip. Larger redder data points correspond to more slip. Smaller, bluer data points are less slip. GTS measurements are the kite-shaped regions and are colored by thermal inertia. Bluer is lower thermal inertia, redder is higher thermal inertia. Plots are overlaid on top of Hirise orbital imagery with estimated terrain classes from Navcam imagery. Black lines show each slip measurements matched to the best GTS measurements.

as described in Section $\mathrm{V}$-A for slip data. Example matches are shown in Figure 5 In some cases, there is very clear overlap between the slip and thermal inertia measurements (e.g. Figures $5 \mathrm{a}$ and $5 \mathrm{~b}$ ). In other cases, there is no clear measurement in that same sand region. This was the case in Moonlight Valley (on the left of Figure 5c), so slip samples from Moonlight valley were not considered in analysis.

For each slip measurement, an orbital estimate of thermal inertia is also identified using THEMIS data. THEMIS is a thermal emission spectrometer orbiting Mars that measures surface temperature at a resolution of $100 \mathrm{~m}$ per pixel [11]. Thermal inertia estimates were taken from data products produced by the THEMIS team [10]. Thermal inertia for each sample is identified by finding the corresponding pixel in the thermal inertia map. An example is shown in Figure 6.

\section{Resampling}

All of the identified measurements of slip in sand are summarized in Table [ This table illustrates the small amount of independent samples available and the imbalance of the data. There are two main problems: (1) there are only 8 independent regions of sand that Curiosity has driven through and (2) slip measurements are not evenly distributed between sites. This data distribution works if it is assumed that each slip measurement is i.i.d. This is clearly not true since many of these samples are closely related to samples in the same site. A more reasonable assumption is that sand characteristics and resulting slip behavior i.i.d. across the surface. Thus each site should be treated as a sample and not the slip measurement. Underrepresented sites are oversampled so that each of the sites listed in Table $\mathrm{I}$ has the same number of samples.

Because there were few samples at high slopes, four syn- 


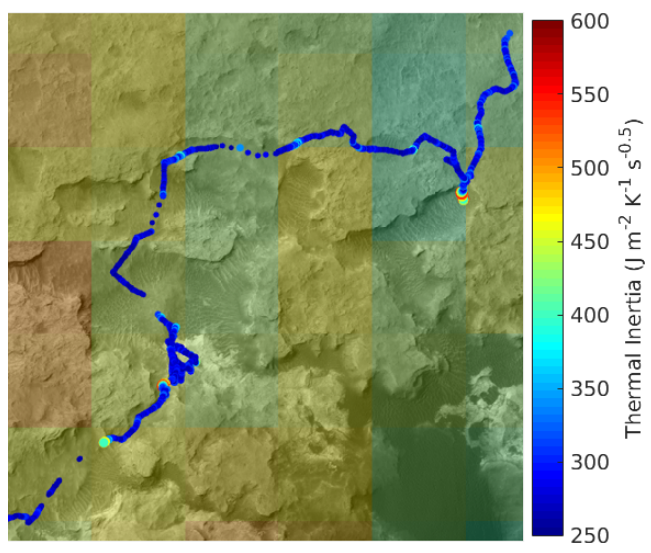

Fig. 6: Orbital THEMIS thermal inertia image overlaid on Hirise camera image. Plotted points show Curiosity's path and are sized and colored by slip. Bluer, smaller points have lower slip. Redder, larger points have higher slip. The colormap corresponds to the overlaid THEMIS image.

TABLE I: Drives in sand by site name, first sol, number of samples, maximum slope $x_{\max }$ (degrees), maximum slip $y_{\max }$ (percentage), average GTS thermal inertia $I_{G T S}$, and average THEMIS thermal inertia $I_{\text {THEMIS. }}$. Units of $I$ are $\frac{J}{m^{2} K \sqrt{s}}$.

\begin{tabular}{lrrrrrr}
\hline Site Name & Sol & Num. & $\mathbf{x}_{\max }$ & $\mathbf{y}_{\max }$ & $\mathbf{I}_{\text {GTS }}$ & $\mathbf{I}_{\text {THEMIS }}$ \\
\hline Moosilauke Valley & 672 & 4 & 3.5 & 79.7 & 20 & 451 \\
Hidden Valley & 709 & 50 & 7.4 & 80.7 & 31 & 413 \\
Logans Pass & 983 & 4 & 9.6 & 75.2 & 47 & 438 \\
Logans Pass & 978 & 4 & 8.2 & 59.8 & 51 & 400 \\
Jubilee Pass & 747 & 5 & 8.4 & 15.4 & 88 & 467 \\
Ripple Crossing & 683 & 11 & 8.4 & 47.9 & 107 & 456 \\
Dingo Gap & 528 & 13 & 13.9 & 46.4 & 108 & 478 \\
Ripple Crossing & 606 & 23 & 8.1 & 33.3 & 109 & 483 \\
Artists Drive & 923 & 21 & 16.4 & 83.4 & 143 & 456 \\
\hline Total: 9 sites & 135 & & & \\
\hline
\end{tabular}

thetic samples were also added for training from the Earthcalibrated model [24]. Samples were added at the minimum slope with $100 \%$ slip, $x_{\max }$, and $-x_{\max }$ with $-100 \%$. For $\mathrm{Cu}-$ riosity, $x_{\max }=18^{\circ}$. These were repeated at both the maximum and minimum thermal inertia (for the given instrument). These constraining points help reduce overfitting to moderate slopes.

\section{RESULTS AND DISCUSSION}

Slip samples are plotted in Figure 7 and colorized by thermal inertia for GTS estimates and THEMIS. The range of thermal inertia estimates are very different between the two sources; however, this is not considered problematic since only within-model comparisons are made [23, 46, 28]. This discrepancy is likely influenced by the use of MER dust opacity estimates and the significantly greater spatial averaging in the orbital data. Qualitatively, both thermal inertia sources distinguish between higher-slip sand and lower-slip sand at a given slope value. In particular, the highest slip data points either occurred at high slope $\left(\sim 15^{\circ}\right)$ and high thermal inertia or at lower slope $\left(\sim 5^{\circ}\right)$ but low thermal inertia. The low slope
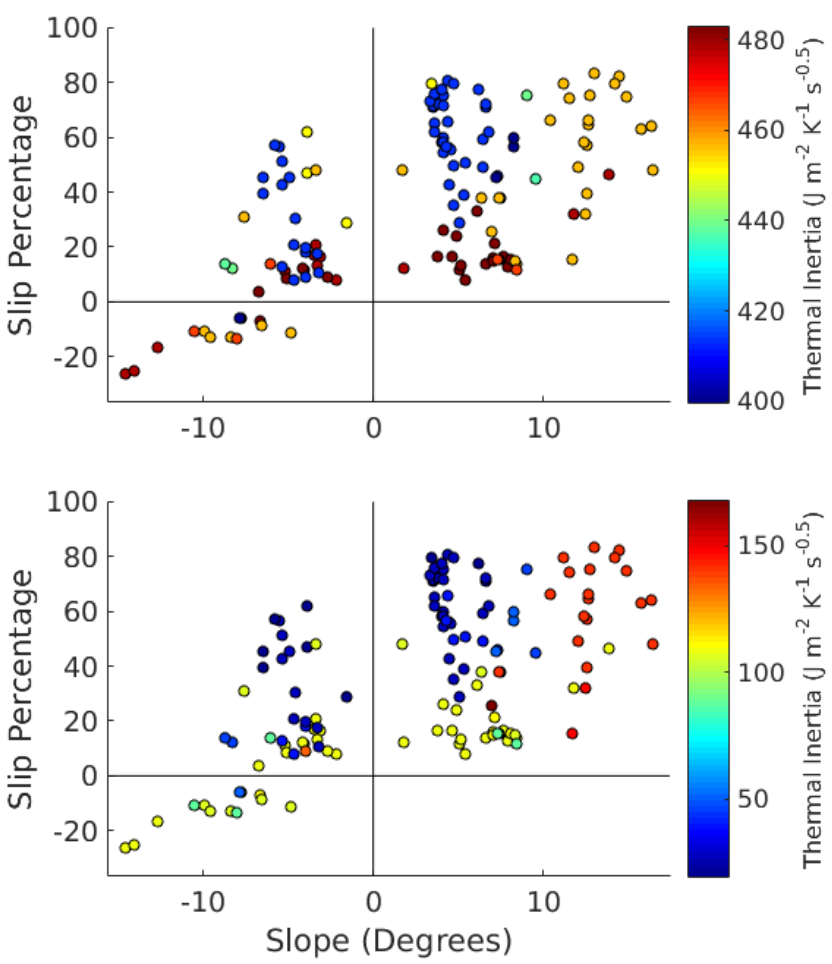

Fig. 7: Slip versus slope data and models for sand colorized by thermal inertia for THEMIS (top) and GTS (bottom).

samples with the highest slip occurred at Moosilauke Valley, Hidden Valley, and Logan's Pass. These three regions also correspond to the GTS lowest thermal inertia.

To quantify the benefit of thermal inertia in slip prediction, three models were compared: (1) a single-expert model, (2) a two-expert model using GTS measurements, and (3) a twoexpert model using THEMIS measurements. The single-expert model does not take into account thermal inertia and learns slip only as a function of slope (Equation 8) for visually classified sand. The models are compared based on their root mean squared error (RMSE) for the best-fit model on all of the data and using leave-one-out cross validation. In this context, "leaving one out" means training on data from $N-1$ sites and testing on the remaining site during each iteration of cross validation. Performance was compared for using both the original and resampled data.

Slip functions for the single-expert and two-expert models using resampled data are shown in Figure 8. The two-expert model shown uses GTS data; however, the THEMIS model was very similar. Overall results are shown in Table II] In all metrics, the two-expert models that used thermal inertia and slope had lower error than the single-expert model that only used slope. This supports the hypothesis that thermal inertia improves slip prediction on Mars. The rover slips more as thermal inertia decreases. With resampling, learned thresholds were 69 and $453 \frac{J}{m^{2} K \sqrt{s}}$ for GTS and THEMIS, respectively.

The degraded performance in the cross validation metrics from the best-fit RMSE suggests that the models are overfitting 


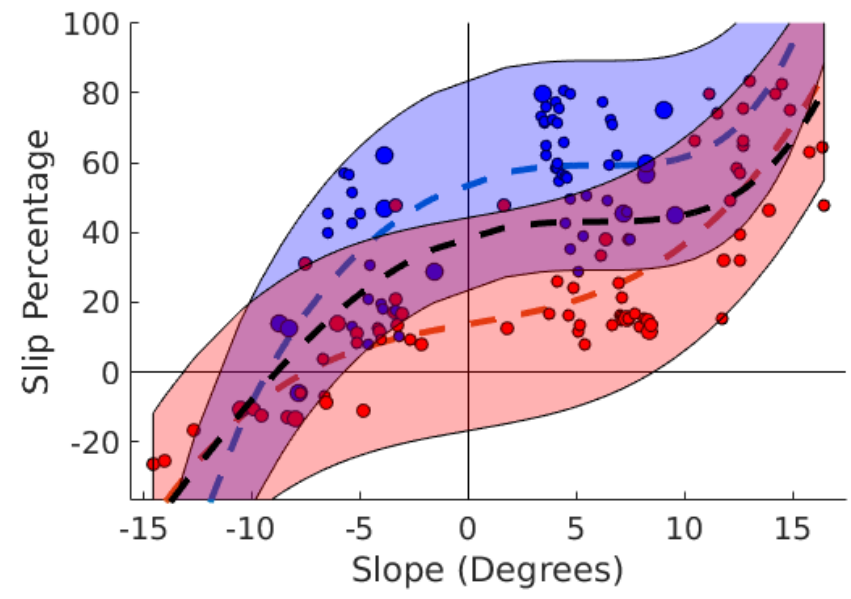

Fig. 8: Slip models for loose sand. Curves for the best-fit two-expert model using GTS data are plotted in red and blue. Mean and two-sigma bounds are plotted for both. Samples are colorized by the most likely expert and sized by relative weight after resampling. The mean of the single-expert model is plotted in black. Models learned from resampled data.

the data. However, this is expected given that there are only 9 unique sites. In cross validation the two-expert models have lower error than the single-expert model showing that models including thermal inertia have higher predictive performance. The reduction in the best-fit RMSE with resampling is expected. More samples are clustered around fewer points. However, the corresponding reduction in cross validation error suggests that it also improves prediction performance.

The higher cross-validation error for the THEMIS model with resampling is mostly caused by misclassifying Moosilauke valley. The THEMIS thermal inertia estimate is higher (relatively) than the GTS estimate (see Table I). Moosilauke valley is in a small ripple field surrounded by high thermal inertia terrain. When THEMIS averages at $100 \mathrm{~m}$ resolution, the result is a higher observed thermal inertia than likely corresponds to the sand alone. Thus, the GTS and THEMIS models agreed more closely for larger areas of sand. This shows the benefit of ground-based sampling. It can identify smaller hazardous regions than lower-resolution orbital instruments. Even the GTS rarely measures a location exactly where the rover is driving. Heterogeneity also makes it difficult to interpret terrain as a single "type". Despite imprecise measurements, thermal inertia improved slip prediction significantly.

There are several factors that affect traversability in sand: rover configuration, sand properties, and surface geometry. The apparent thermal inertia is affected by the physical properties of the sand grains and depth of the surface layer. Without ground truth (e.g. for sand depth), it is difficult to determine exactly how much each factor affected either the thermal inertia measurements or the wheel-terrain interaction. However, evidence suggests both depth and physical properties were important. For example, Arvidson et al. [4] estimate that the properties of the sand at Dingo Gap were more traversable
TABLE II: Root mean squared error (RMSE) for the best-fit model using all data and leave-one-out cross validation (CV) for all models both with and without resampling.

\begin{tabular}{lrrrrrr}
\hline \multirow{2}{*}{ Model } & \multicolumn{2}{c}{ No Resampling } & & \multicolumn{2}{c}{ Resampling } \\
\cline { 2 - 3 } \cline { 6 - 7 } & Best-fit & CV & & Best-fit & CV \\
\hline Single Expert (Slope Only) & 22.0 & 30.3 & & 20.5 & 24.7 \\
Two-Expert THEMIS & $\mathbf{1 5 . 9}$ & 22.7 & & $\mathbf{1 5 . 0}$ & 24.4 \\
Two-Expert GTS & 16.8 & $\mathbf{2 0 . 4}$ & & 15.1 & $\mathbf{1 8 . 6}$ \\
\hline
\end{tabular}

than at Hidden Valley. Higher GTS thermal inertia at Dingo Gap supports this claim. In addition, on visual inspection, sand depth is related to both traversability and thermal inertia. Sand at Artist's Drive (Figure 4d) appears shallower than other areas such as Hidden Valley (Figure 4b). It had correspondingly higher thermal inertia and the rover slipped at higher slopes.

\section{CONCLUSIONS}

This paper presented an approach for improving slip prediction on Mars by classifying sand using thermal inertia measurements. Slip prediction accuracy was compared among a single-expert, vision-only model and two-expert models using both GTS- and THEMIS-based thermal inertia measurements. Results showed that separating low-slip, high thermal inertia sand from high-slip, low thermal inertia sand significantly improved accuracy. Slip predictions from orbital and groundbased data were similar. However, GTS measurements more reliably detected smaller regions of hazardous sand. Resampling data by assuming that regions of sand rather than slip measurements were i.i.d. also improved prediction accuracy.

Given this encouraging empirical evidence and the theoretical connection, there is little doubt a correlation exists between thermal inertia and mobility for rovers operating in granular materials on Mars. However, the terrain on Mars is very diverse and Curiosity's data is limited to measurements in sand from one region of Mars. More data must be gathered to determine whether this holds true in other types of terrain (e.g. duricrust over sand). Before considering implementation, it is necessary to fully understand what types of hazards can and can't be detected, at what times of day, how many of those hazards couldn't be detected visually, and how rover operations would have to be adjusted to account for thermal inertia measurements. Further analysis should also include effects of heterogeneous terrain and ripples in sand, which were likely causes of intraclass variance.

\section{ACKNOWLEDGMENTS}

The authors wish to thank Mark Maimone, Sylvain Piqueux, Masahiro Ono, Jeng Yen, and Ray Arvidson for their help and advice. We thank the PDS Geosciences Node for providing image and thermal data. We also thank the Mars Science Laboratory team for providing the slip data that enabled this investigation. Portions of this research were carried out at the Jet Propulsion Laboratory, California Institute of Technology, under a contract with NASA. This work was supported by a NASA Space Technology Research Fellowship. 


\section{REFERENCES}

[1] Anelia Angelova, Larry Matthies, Daniel Helmick, and Pietro Perona. Slip Prediction Using Visual Information. In Proceedings of Robotics: Science and Systems, Philadelphia, 2006.

[2] Anelia Angelova, Larry Matthies, Daniel Helmick, and Pietro Perona. Learning and Prediction of Slip from Visual Information. Journal of Field Robotics, 24(3): 205-231, 2007.

[3] R. E. Arvidson, J. F. Bell, P. Bellutta, N. A. Cabrol, J. G. Catalano, J. Cohen, L. S. Crumpler, D. J. Des Marais, T. A. Estlin, W. H. Farrand, R. Gellert, J. A. Grant, R. N. Greenberger, E. A. Guinness, K. E. Herkenhoff, J. A. Herman, K. D. Iagnemma, J. R. Johnson, G. Klingelhöfer, R. Li, K. A. Lichtenberg, S. A. Maxwell, D. W. Ming, R. V. Morris, M. S. Rice, S. W. Ruff, A. Shaw, K. L. Siebach, P. A. De Souza, A. W. Stroupe, S. W. Squyres, R. J. Sullivan, K. P. Talley, J. A. Townsend, A. Wang, J. R. Wright, and A. S. Yen. Spirit Mars Rover Mission: Overview and Selected Results from the Northern Home Plate Winter Haven to the Side of Scamander crater. Journal of Geophysical Research: Planets, 115(9):1-19, 2010.

[4] Raymond E. Arvidson, Karl D. Iagnemma, Mark Maimone, Abigail A. Fraeman, Feng Zhou, Matthew C. Heverly, Paolo Bellutta, David Rubin, Nathan T. Stein, John P. Grotzinger, and Ashwin R. Vasavada. Mars Science Laboratory Curiosity Rover Megaripple Crossings up to Sol 710 in Gale Crater. Journal of Field Robotics, 2016.

[5] Joshua L. Bandfield, Eugenie Song, Paul O. Hayne, Brittany D. Brand, Rebecca R. Ghent, Ashwin R. Vasavada, and David A. Paige. Lunar Cold Spots: Granular Flow Features and Extensive Insulating Materials Surrounding Young Craters. Icarus, 231:221-231, 2014.

[6] Christopher M. Bishop. Pattern Recognition And Machine Learning. 2006.

[7] Christopher A. Brooks and Karl Iagnemma. SelfSupervised Terrain Classification for Planetary Surface Exploration Rovers. Journal of Field Robotics, 29(3): 445-468, 2012.

[8] W. David Carrier. The Four Things You Need to Know about the Geotechnical Properties of Lunar Soil Lunar Geotechnical Institute, (September):23, 2005.

[9] S. Chhaniyara, C. Brunskill, B. Yeomans, M. C. Matthews, C. Saaj, S. Ransom, and L. Richter. Terrain Trafficability analysis and Soil Mechanical Property Identification for Planetary Rovers: A Survey, Journal of Terramechanics, 49(2):115-128, Apr 2012.

[10] P R Christensen, R L Fergason, C S Edwards, and J Hill. THEMIS-Derived Thermal Inertia Mosaic of Mars: Product Description and Science Results 44th Lunar and Planetary Science Conference, page Abstract \#2822, 2013.

[11] Philip R Christensen, Bruce M Jakosky, Hugh H Kieffer,
Michael C Malin, Harry Y Mcsween, Kenneth Nealson, Greg L Mehall, Steven H Silverman, Steven Ferry, Michael Caplinger, and Michael Ravine. The Thermal Emission System (THEMIS) for the Mars 2001 Odyssey Mission. Space Science Reviews, 110:85-130, 2004.

[12] Christopher Cunningham, Issa Nesnas, and William L Whittaker. Terrain Traversability Prediction by Imaging Thermal Transients. In IEEE Conference on Robotics and Automation, pages 3947-3952, Seattle, 2015.

[13] Christopher Cunningham, Uland Wong, Kevin M. Peterson, and William L. Red Whittaker. Predicting Terrain Traversability from Thermal Diffusivity. In Field and Service Robotics, volume 105, pages 61-74, Brisbane, Australia, 2015.

[14] Christopher Cunningham, William L. Whittaker, and Issa Nesnas. Detecting Loose Regolith in Lunar Craters Using Thermal Imaging. In ASCE Conference on Earth and Space, 2016.

[15] Christopher Cunningham, Masahiro Ono, Issa Nesnas, Jeng Yen, and William L. Whittaker. Locally-Adaptive Slip Prediction for Planetary Rovers Using Gaussian Processes In IEEE Conference on Robotics and Automation, 2017.

[16] R. L. Fergason, P. R. Christensen, M. P. Golombek, and T. J. Parker. Surface Properties of the Mars Science Laboratory Candidate Landing Sites: Characterization from Orbit and Predictions Space Science Reviews, 170 (1-4):739-773, May 2012.

[17] Robin L. Fergason, Philip R. Christensen, James F. Bell, Matthew P. Golombek, Kenneth E. Herkenhoff, and Hugh H. Kieffer. Physical Properties of the Mars Exploration Rover Landing Sites as Inferred from MiniTES-Derived Thermal Inertia Journal of Geophysical Research: Planets, 111(2):E02S21, 2006.

[18] Robin L. Fergason, Philip R. Christensen, and Hugh H. Kieffer. High-Resolution Thermal Inertia Derived from the Thermal Emission Imaging System (THEMIS): Thermal Model and Applications Journal of Geophysical Research: Planets, 111(12):1-22, 2006.

[19] Steven B. Goldberg, Mark W. Maimone, and Lany Matthies. Stereo Vision and Rover Navigation Software for Planetary Exploration. In IEEE Aerospace Conference Proceedings, volume 5, pages 2025-2036, 2002.

[20] M. Golombek, J. Grant, D. Kipp, A. Vasavada, R. Kirk, R. Fergason, P. Bellutta, F. Calef, K. Larsen, Y. Katayama, A. Huertas, R. Beyer, A. Chen, T. Parker, B. Pollard, S. Lee, Y. Sun, R. Hoover, H. Sladek, J. Grotzinger, R. Welch, E. Noe Dobrea, J. Michalski, and M. Watkins. Selection of the Mars Science Laboratory Landing Site Space Science Reviews, 170(1-4):641-737, 2012.

[21] John P. Grotzinger, Joy Crisp, Ashwin R. Vasavada, Robert C. Anderson, Charles J. Baker, Robert Barry, David F. Blake, Pamela Conrad, Kenneth S. Edgett, Bobak Ferdowski, Ralf Gellert, John B. Gilbert, Matt Golombek, Javier Gómez-Elvira, Donald M. Hassler, 
Louise Jandura, Maxim Litvak, Paul Mahaffy, Justin Maki, Michael Meyer, Michael C. Malin, Igor Mitrofanov, John J. Simmonds, David Vaniman, Richard V. Welch, and Roger C. Wiens. Mars Science Laboratory Mission and Science Investigation, volume 170. 2012.

[22] Ibrahim Halatci, Christopher A. Brooks, and Karl Iagnemma. Terrain Classification and Classifier Fusion for Planetary Exploration Rovers In IEEE Aerospace Conference Proceedings, pages 1-11. Ieee, 2007.

[23] Victoria E. Hamilton, Ashwin R. Vasavada, Eduardo Sebastiiann, Manuel De La Torre Juarez, Miguel Ramos, Carlos Armiens, Raymond E. Arvidson, Isaias Carrasco, Philip R. Christensen, Miguel A. De Pablo, Walter Goetz, Javier Gomez-Elvira, Mark T. Lemmon, Morten B. Madsen, F. Javier Martin-Torres, Jesus Martinez-Frias, Antonio Molina, Marisa C. Palucis, Scot C R Rafkin, Mark I. Richardson, R. Aileen Yingst, and Maria Paz Zorzano. Observations and Preliminary Science Results from the First 100 Sols of MSL Rover Environmental Monitoring Station Ground Temperature Sensor Measurements at Gale Crater. Journal of Geophysical Research: Planets, 119(4):745-770, 2014.

[24] Matt Heverly, Jaret Matthews, Justin Lin, Dan Fuller, Mark Maimone, Jeffrey Biesiadecki, and John Leichty. Traverse Performance Characterization for the Mars Science Laboratory Rover. Journal of Field Robotics, 30 (6):835-846, 2013.

[25] Ken Ho, Thierry Peynot, and Salah Sukkarieh. Nonparametric Traversability Estimation in Partially Occluded and Deformable Terrain 2016.

[26] Ogbonnaya Igwe, Kyoji Sassa, and Fawu Wang. The Influence of Grading on the Shear Strength of Loose Sands in Stress-Controlled Ring Shear Tests. Landslides, 4(1):43-51, 2007.

[27] Bruce M. Jakosky. On the Thermal Properties of Martian Fines Icarus, 66(1):117-124, 1986.

[28] Hugh H. Kieffer. Thermal Model for Analysis of Mars Infrared Mapping, Journal of Geophysical Research: Planets, 118(3):451-470, 2013.

[29] Poul V. Lade and Daniel D Overton. Cementation Effects in Frictional Materials. Journal of Geotechnical Engineering, 115(10):1373-1387, 1989.

[30] Mark T. Lemmon. The Mars Science Laboratory Optical Depth Record In LPI Contributions, pages 1-2, 2014.

[31] Mark T. Lemmon, Michael J. Wolff, James F. Bell, Michael D. Smith, Bruce A. Cantor, and Peter H. Smith. Dust Aerosol, Clouds, and the Atmospheric Optical Depth Record Over 5 Mars Years of the Mars Exploration Rover Mission. Icarus, 251:96-111, 2015.

[32] Mark Maimone. No A Martian Vision: Impact of JPL Robotics Vision and Mobility Research on the Mars Rovers In JPL Robotics Section Senior Lecture Series, 2016.

[33] Mark Maimone, Yang Cheng, and Larry Matthies. Two Years of Visual Odometry on the Mars Exploration Rovers Journal of Field Robotics, 24(3):169-186, 2007.
[34] G. M. Martínez, N. Rennõ, E. Fischer, C. S. Borlina, B. Hallet, M. De La Torre Juárez, A. R. Vasavada, M. Ramos, V. Hamilton, J. Gomez-Elvira, and R. M. Haberle. Surface Energy Budget and Thermal Inertia at Gale Crater: Calculations from Ground-Based Measurements Journal of Geophysical Research: Planets, 119 (8):1822-1838, 2014.

[35] M T Mellon, B M Jakosky, $\mathrm{H}$ H Kieffer, and P R Christensen. High-Resolution Thermal Inertia Mapping from the Mars Global Surveyor Thermal Emission Spectrometer Icarus, 148(2):437-455, Dec 2000.

[36] Julia K. Morgan. Numerical Simulations of Granular Shear Zones Using the Distinct Element Method 2. Effects of Particle Size Distribution and Interparticle Friction on Mechanical Behavior. Journal of Geophysical Research, 104(B2):2721-2732, 1999.

[37] Kyohei Otsu, Masahiro Ono, Thomas J. Fuchs, Ian Baldwin, and Takashi Kubota. Autonomous Terrain Classification With Co- and Self-Training Approach. IEEE Robotics and Automation Letters, 1(2):814-819, 2016.

[38] Howard A. Perko, John D. Nelson, and Jacklyn R. Green. Mars Soil Mechanical Properties and Suitability of Mars Soil Simulants Journal of Aerospace Engineering, 19 (July):169-176, 2006.

[39] S. Piqueux and P. R. Christensen. A Model of Thermal Conductivity for Planetary Soils: 2. Theory for Unconsolidated Soils Journal of Geophysical Research: Planets, 114(9):1-20, 2009.

[40] S. Piqueux and P. R. Christensen. A Model of Thermal Conductivity for Planetary Soils: 2. Theory for Cemented Soils Journal of Geophysical Research: Planets, 114(9): 1-20, 2009.

[41] M A Presley and P R Christensen. Thermal Conductivity Measurements of Particulate Materials: 5. Effect of Bulk density and Particle Shape. Journal of Geophysical Research: Planets, 115(E7):13, jul 2010.

[42] Marsha A Presley and Philip R Christensen. Thermal Conductivity Measurements of Particulate Materials 2. Results. Journal of Geophysical Research: Planets, 102 (E3):6551-6566, 1997.

[43] John C. Price. Thermal Inertia Mapping: A New View of the Earth Journal of Geophysical Research, 82(18): 2582, 1977.

[44] Nathaniel E. Putzig. Thermal Inertia and Surface Heterogeneity on Mars PhD thesis, University of Colorado, 2006.

[45] Nathaniel E. Putzig and Michael T. Mellon. Apparent Thermal Inertia and the Surface Heterogeneity of Mars Icarus, 191(1):68-94, 2007.

[46] N.E. Putzig, M.T. Mellon, B.M. Jakosky, S.M. Pelkey, S. Martínez-Alonso, B.M. Hynek, and N.W. Murphy. Mars Thermal Inertia from THEMIS Data Lunar and Planetary Science Conference, 35:1863, 2004.

[47] Carl Edward Rasmussen and Zoubin Ghahramani. Infinite Mixtures of Gaussian Process Experts Advances in Neural Information Processing Systems, 2:881-888, 
2002.

[48] Brandon Rothrock, Jeremie Papon, Ryan Kennedy, Masahiro Ono, Matt Heverly, and Christopher Cunningham. SPOC : Deep Learning-based Terrain Classification for Mars Rover Missions. In AIAA: Space, 2016.

[49] Eduardo Sebastián, Carlos Armiens, Javier GómezElvira, María P. Zorzano, Jesus Martinez-Frias, Blanca Esteban, and Miguel Ramos. The Rover Environmental Monitoring Station Ground Temperature Sensor: A Pyrometer for Measuring Ground Temperature on Mars. Sensors, 10(10):9211-9231, 2010.

[50] S. W. Squyres, R. E. Arvidson, D. Bollen, James F. Bell, J. Bruckner, N. A. Cabrol, W. M. Calvin, M. H. Carr, Philip R. Christensen, B. C. Clark, L. Crumpler, D. J. Des Marais, C. D'Uston, T. Economou, J. Farmer, W. H. Farrand, W. Folkner, R. Gellert, Timothy D. Glotch, Matthew P. Golombek, S. Gorevan, J. A. Grant, R. Greeley, J. Grotzinger, K. E. Herkenhoff, S. Hviid, Jeffrey R. Johnson, G. Klingelhofer, A. H. Knoll, G. Landis, Mark T. Lemmon, R. Li, M. B. Madsen, M. C. Malin, S. M. McLennan, H. Y. McSween, D. W. Ming, J. Moersch, R. V. Morris, T. Parker, Jr W. Rice, L. Richter, R. Rieder, C. Schroder, M. Sims, M. Smith, P. Smith, L. A. Soderblom, R. J. Sullivan, N. J. Tosca, H. Wanke, T. Wdowiak, Michael J. Wolff, and Albert S. Yen. Overview of the Opportunity Mars Exploration Rover Mission to Meridiani Planum: Eagle Crater to Purgatory Ripple. Journal of Geophysical Research: Planets, 111 (12):1-19, 2006.

[51] Ashwin R. Vasavada, Joshua L. Bandfield, Benjamin T. Greenhagen, Paul O. Hayne, Matthew A. Siegler, Jean Pierre Williams, and David A. Paige. Lunar Equatorial Surface Temperatures and Regolith Properties from the Diviner Lunar Radiometer Experiment Journal of Geophysical Research: Planets, 117(4):1-12, Dec 2012.

[52] Li Chang Wang, Wei Long, and Shi Juan Gao. Effect of Moisture Content, Void Ratio and Compacted Sand Content on the Shear Strength of Remolded Unsaturated Clay. Electronic Journal of Geotechnical Engineering, 19(Q):4413-4426, 2014.

[53] Anwar EZ Wissa, Charles Cushing Ladd, and T. William Lambe. Effetive Stress Strength Parameters of Stabilized Soils. In MIT Department of Civil Engineering, 1964.

[54] J. Y. Wong. Predicting the Performances of Rigid Rover Wheels on Extraterrestrial Surfaces Based on Test Results Obtained on Earth Journal of Terramechanics, 49(1):49-61, 2012.

[55] Seniha Esen Yuksel, Joseph N. Wilson, and Paul D. Gader. Twenty Years of Mixture of Experts. IEEE Transactions on Neural Networks and Learning Systems, 23(8):1177-1193, 2012. 